\title{
COMPARATIVE IMAGING STUDY IN ULTRASOUND, MRI, CT AND DSA USING A MULTI-MODALITY RENAL ARTERY PHANTOM
}

\author{
DEIRDRE M. KING*, ANDREW J. FAGAN $\dagger$, CARMEL M. MORAN $\ddagger$, AND \\ JACINTA E. BROWNE*
}

*Medical Ultrasound Physics and Technology Group, School of Physics, Dublin Institute of Technology, Dublin 8, Ireland,

$\dagger$ Centre for Advanced Medical Imaging (CAMI), St James's Hospital, Dublin 8, Ireland and

†Department of Medical Physics, University of Edinburgh, Edinburgh, UK

\begin{abstract}
Purpose: A range of anatomically-realistic multi-modality renal artery phantoms consisting of vessels with varying degrees of stenosis was developed and evaluated using four imaging techniques currently used to detect renal artery stenosis (RAS). The spatial resolution required to visualize vascular geometry and the velocity detection performance required to adequately characterize blood flow in patients suffering from RAS is currently ill-defined, with the result that no one imaging modality has emerged as a gold standard technique for screening for this disease.
\end{abstract}

Methods: The phantoms, which contained a range of stenosis values $(0,30,50,70$ and $85 \%$ ), were designed for use with ultrasound, magnetic resonance imaging, X-ray computed tomography and X-ray digital subtraction angiography. The construction materials used were optimized with respect to their ultrasonic speed of sound and attenuation coefficient, MR relaxometry $\left(\mathrm{T}_{1}, \mathrm{~T}_{2}\right)$ properties, and Hounsfield number / X-ray attenuation coefficient, with a design capable of tolerating high-pressure pulsatile flow. Fiducial targets, incorporated into the phantoms to allow for 
registration of images among modalities, were chosen to minimize geometric distortions.

Results: High quality distortion-free images of the phantoms with good contrast between vessel lumen, fiducial markers and background tissue to visualize all stenoses were obtained with each modality. Quantitative assessments of the grade of stenosis revealed significant discrepancies between modalities, with each underestimating the stenosis severity for the higher-stenosed phantoms $(70 \%$ and $85 \%$ ) by up to $14 \%$, with the greatest discrepancy attributable to DSA.

Conclusions: The design and construction of a range of anatomically-realistic renal artery phantoms containing varying degrees of stenosis is described. Images obtained using the main four diagnostic techniques used to detect RAS were free from artifacts and exhibited adequate contrast to allow for quantitative measurements of the degree of stenosis in each phantom. Such multi-modality phantoms may prove useful in evaluating current and emerging US, MRI, CT and DSA technology.

Key words - Ultrasound, multi-modality, phantom, in vitro experimentation 


\section{INTRODUCTION}

The renal system plays an important role in maintaining the health of individuals, for example through the removal of waste products from the blood and the controlling of blood pressure and the manufacture of red blood cells. A renal artery stenosis (RAS) can result in clinical syndromes such as renovascular hypertension, ischemic nephropathy and pulmonary edema $[1,2]$, and is associated with an increased risk of cardiovascular mortality. The vast majority of stenoses are caused by atherosclerosis, a common progressive problem that increases in prevalence with age [2] and it is recognized as an important cause of secondary hypertension which afflicts approximately 60 million people in the USA alone [3, 4]. Current evidence also suggests that the presence of atherosclerotic plaque in the renal artery is indicative of the presence of plaque at other sites in the body such as the carotid artery or the coronary artery, which further adds to the cardiovascular risk and long-term prognosis for patients with RAS [5]. Thus, there is a considerable advantage in detecting RAS at the earliest possible stage of the disease, in order to maximize the therapeutic outcome following drug administration or renal vascularisation.

Consequently, there is much clinical interest in the initiation process and progression of atherosclerotic disease in the renal artery, the relationship between stenosis,

25 hypertension and end-stage renal failure, and whether more accurate stenosis assessment techniques would improve patient outcome. The medical imaging techniques currently available to investigate this condition are ultrasound (US), X-ray computed tomography (CT), magnetic resonance imaging (MRI) and X-ray digital subtraction angiography (DSA). However, criteria for selecting the most suitable 
technique for screening patients with suspicion of RAS are currently ill-defined, in terms of the required spatial resolution and velocity detection performance for quantification of the vascular geometry and the characterization of the blood flow. Furthermore, only a limited quantitative assessment of the detection capabilities of these imaging modalities has been carried out to establish the optimum modality or

35 combination of modalities for the diagnosis of renal artery stenosis, in large part due to the lack of a suitable multimodality phantom.

Before a medical imaging technique can be introduced to routine clinical use as a new imaging alternative to the previous gold standard, it must first be validated in order to 40 determine its accuracy, a process usually carried out via expensive and lengthy clinical trials [6]. Such clinical trials often have complicated ethical issues associated with them, experience difficulties recruiting a sufficiently large number of patients which would be required to achieve statistical significance between the performance of the new and existing gold standard techniques. Furthermore, the clinical truth is 45 seldom known, so it is difficult to assess the absolute accuracy of each of these imaging techniques.

However, the validation procedures for new imaging techniques can also include in vitro flow phantom experimentation [7-10] wherein a valid comparative study 50 could be conducted by imaging a standardized vascular phantom with each modality, with the phantom ideally containing fiducial markers to aid image registration. Four such multi-modality vascular phantoms have been developed and described in the literature, each of which contained fiducial markers to aid image registration [7-9, 11]. Frayne et al. developed a carotid vascular phantom for comparative studies of X- 
ray DSA, US and MRI [9]. However, the vessel mimicking material, polyester resin, used for the vessel wall in this phantom was found to be only suitable for X-ray imaging, with artifacts occurring in both US and MR images. Furthermore, the authors used lead core fishing line $(0.8 \mathrm{~mm}$ outer diameter with $0.2 \mathrm{~mm}$ diameter lead core) as the fiducial marking system; this marker system was only clearly detectable

60 in the X-ray images. The approach adopted by Dabrowski et al. used a formaldehydefixed section of a real human iliac artery cannulated onto two acrylic tubes to mimic the vessel wall, and three imaging modalities (DSA, CT, and 3-D B-mode US) were evaluated by comparing images of this phantom [8]. Although the use of ex vivo human vessels assured accurate geometric and tissue properties for this phantom, the 65 use of ex vivo human tissue inherently reduces the lifespan of such phantoms and further renders it impossible to carry out longitudinal or inter-institutional studies. An additional problem that was encountered with this phantom stemmed from the use of small stainless steel ball bearings for the fiducial markers, which introduced significant artifacts in both the US and CT images and would further pose 70 considerable problems for MR imaging. Cloutier et al. developed a multi-modality phantom containing a straight lumen with a double stenosis (70\% and $85 \%)$ [7]; however, the low acoustic velocity compared to in vivo tissue of the agar-oil mixture used in this phantom caused geometric distortion in the ultrasound images, while the latex used to mimic the vessel wall caused a slight reduction in the measurement of

75 the vessel lumen diameter. Although this phantom incorporated two grades of stenosis, the positioning of the stenoses along a straight lumen did not replicate the complexity of a clinical presentation of RAS, where stenoses typically occur near bends in vessels where turbulent flow effects lead to mechanical wall stresses and regions of slow flow conducive to plaque formation/deposition. More recently, 
80 Allard et al. incorporated the use of an alternative to low melting point metal, Isomalt (which is a commercial sugar alcohol), for the fabrication of the vessel geometries [11]. This phantom also used the same layout of fiducial markers as Cloutier et al., however volume shrinkage was observed during the cooling phase for this material which may have affected the final lumen dimensions. There is a clear need for the 85 development of an anatomically-realistic phantom which can be easily and reproducibly manufactured and imaged in a distortion-less manner with each modality, while also incorporating features of disease progression such as vessel stenoses.

90 An alternative approach to building physical models is to use the technique of rapid prototyping, wherein a structure of interest is constructed layer by layer from a 3D computer model of the structure. Using rapid prototyping, it is possible to rapidly and reproducibly produce models with complex shapes, which makes the technique particularly attractive for developing anatomically-realistic phantoms. In the current

95 work, the renal artery morphology was modeled using a 3D dataset from a CT scan of a patient imported into a computer-aided design (CAD) software package, from which a physical model of the artery was produced using rapid prototyping. This model was then used to produce anatomically-realistic renal artery flow phantoms with in-built fiducial markers, using customized materials which optimally mimicked tissue 100 properties with respect to ultrasound, MRI and X-ray DSA/CT. Attention was focused on ensuring compatibility with each modality, from both a safety perspective (for example, no ferromagnetic materials were used) and also the ability to produce artifact-free images with adequate image contrast with each modality. In addition, a range of stenosis values $(0,30,50,70,85 \%)$ were introduced into the phantoms, in 
order to investigate the usefulness of the phantoms in assessing the geometric accuracy of each modality. The aim of the work was thus to develop a suitable RASmimicking phantom and demonstrate its potential in a comparative studies involving US, MRI, CT and DSA.

110

\section{METHODS AND MATERIALS}

\section{TMM characterization}

An agar based tissue mimicking material (TMM) developed by Terlinck et al. (1998) [12] was chosen for the phantoms, the constituents of which are give in Table 1, and its manufacture is described elsewhere [13]. The acoustic properties, in particular speed of sound and attenuation coefficient were verified using a Scanning Acoustic Macroscope with a broadband transducer centered at 2.25 MHz (Ultrasonic Sciences Limited, Fleet, UK) using the pulse-echo substitution technique [14]. To verify the MR properties of the TMM, $T_{1}$ and $T_{2}$ relaxometry measurements were carried out using a two-point ratio method with a mixed sequence implemented on a $3 \mathrm{~T}$ Philips Achieva MRI System (Philips Medical Systems, Netherlands), wherein inversion recovery (IR) and spin echo (SE) pulse sequence modules were consecutively combined in one cycle to provide a measure of $T_{1}$ and $T_{2}$. This technique is insensitive to phase errors due to the use of a phase correction built into the sequence. The SE and IR repetition time (TR) values used for the image acquisition were 6000 and 7000 ms respectively, chosen to allow for a full recovery of magnetisation in each case, while the IR delay time was set at $800 \mathrm{~ms}$. The $T_{2}$ measurements were carried out using a Carr-Purcell Meiboom-Gill sequence module with eight echoes from $10-80 \mathrm{~ms}$ in increments of $10 \mathrm{~ms}$. The Hounsfield (CT) number was 
determined from an image of the TMM acquired using a 64-slice Sensation (Siemens

135 Medical Solutions, Karlsruhe, Germany), CT scanner at $120 \mathrm{kVp}$. Although it was not possible to accurately measure the X-ray attenuation coefficient of the TMM (as a mono-energetic X-ray source was not available), a transmission comparison was carried out between the TMM and solid water phantoms using a standard X-ray imaging system (Digital Diagnostic system, Philips Medical Systems, The

140 Netherlands) and a radiation detector (Model 9010 Radiation Monitor Controller with 6cc ionisation chamber, Radcal Corp., USA). Measurements were performed at multiple $\mathrm{kVp}$ settings (50, 60, 81, 102 and $125 \mathrm{kVp}$ ) using $\mathrm{TMM}$ and water thicknesses of $5 \mathrm{~cm}$.

Table 1 Weight composition of the TMM

\begin{tabular}{l|c}
\hline \multicolumn{1}{c|}{ Component } & Weight composition (\%) \\
\hline Distilled water & 82.97 \\
Glycerol & 11.21 \\
Silicon carbide (400 grain) & 0.53 \\
Aluminium oxide $(3 \mu \mathrm{m})$ & 0.94 \\
Aluminium oxide $(0.3 \mu \mathrm{m})$ & 0.88 \\
Benzalkoniumchloride & 0.46 \\
Agar & 3.0 \\
\hline \hline
\end{tabular}

\section{Phantom construction}

150 To develop an anatomically-realistic renal artery flow phantom, a CAD-based model of a healthy renal artery was developed from a clinical 3D CT dataset. Ethics approval was obtained from Dublin Institute of Technology's Research Ethics Committee. The CT images were imported into a commercial software package (MIMICS 10, Materialise, Leuven, Belgium) which provided an interface for the 155 visualization and segmentation of $\mathrm{CT}$ images and the $3 \mathrm{D}$ rendering of a region of 
interest (ROI). The computer model of the renal artery was exported from SOLID WORKS into the Rapid Prototyping machine, a $\mathrm{Z}$ printer 310 Plus 3D printer (Zcorporation, MA, USA), in the form of STL files from which a physical model of the artery was produced by rapid prototyping. The CAD computer model used to 160 produce the healthy artery, which had a diameter of $6.8 \mathrm{~mm}$, is presented in Figure 1. The physical model thus produced was then used to create a master silicone negative mould, into which a low melting point alloy (melting point: $47^{\circ} \mathrm{C}$, MCP 47 , Mining and Chemical Products Ltd., Northamptonshire, UK) was poured. Once the metal core had set, it was removed from the silicone mould and positioned within the phantom 165 container and the liquid-phase TMM was poured around it and in turn allowed to set. The metal core was subsequently removed through melting by heating the phantoms to $50^{\circ} \mathrm{C} \leq \mathrm{T} \leq 60^{\circ} \mathrm{C}$, which is in excess of the melting temperature of the metal alloy. In this way, it was possible to create a lumen in the TMM with an anatomicallyrealistic geometry.

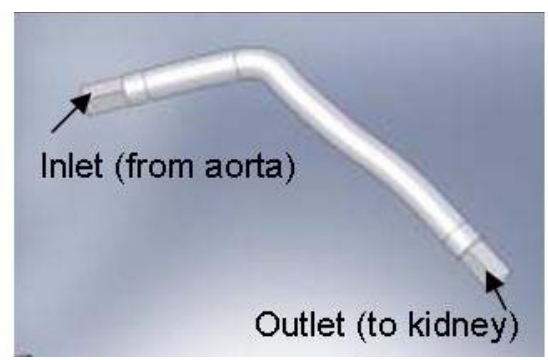

Figure 1 Normal renal artery computer model

In addition to the healthy artery (i.e. with no stenosis), a range of other arteries were produced with stenoses of $30,50,70$ and $85 \%$. These were produced by using a series of symmetrical inserts for each grade of stenosis, each of which was $10 \mathrm{~mm}$ in length (Figure 2). These inserts were placed in the master silicon negative mould before the liquid metal alloy was poured, and hence the shape of the resultant metal 
cores, which were used to create the vessel lumen, already contained the shape of the different degrees of stenoses. To ensure the entire metal alloy was removed from the stenosed phantoms, the metal core was wrapped in cling film prior to its placement in the phantom container and the addition of the TMM. Thus, when the phantom was heated, the entire liquefied metal alloy was removed together with the cling film [15]. Following discussions with an experienced vascular clinical measurement scientist and a review of the literature, the position of the stenoses was chosen to lie approximately $10 \mathrm{~mm}$ from aorta which corresponds to $10 \mathrm{~mm}$ from the inlet of the model as it has been shown that more than $90 \%$ of stenoses are caused by atherosclerosis, which typically occur at the renal artery origin [9]. Additional information regarding the manufacture of these phantoms is provided elsewhere [15].

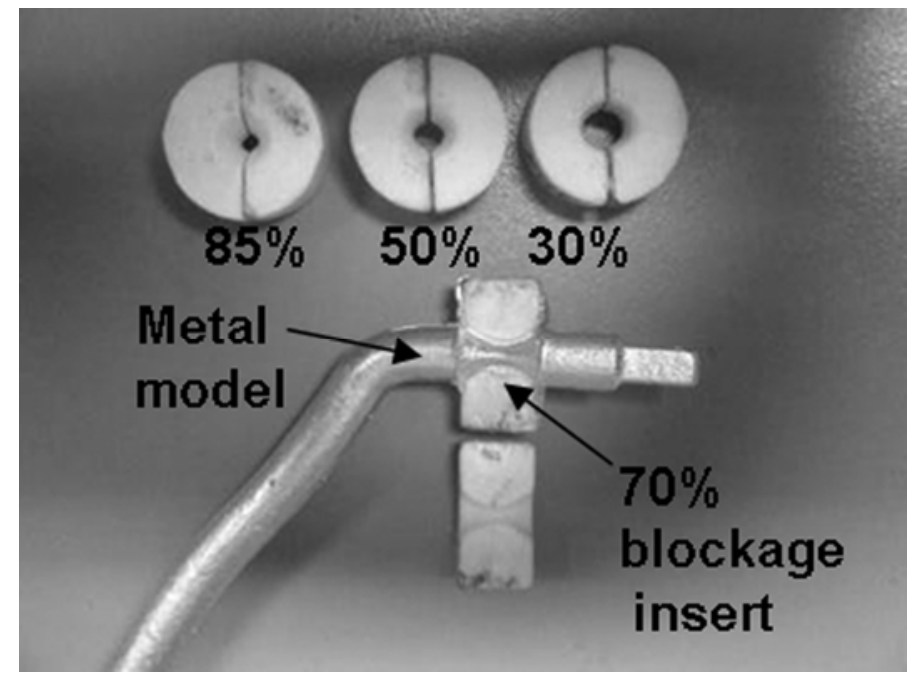

Figure 2 Removal inserts used to fabricate the diseased models of the renal artery. Reproduced with permission [15]

195 In addition to providing a realistic comparison of the imaging modalities, it was important to ensure that the image acquisition parameters used in each case reflected those used clinically. Such parameters are often determined automatically by the 
imaging systems, for example MRI uses automatic pre-scan calibration procedures to determine detector coil loading, radiofrequency power and detector gain levels, while X-ray DSA systems use feed-back mechanisms such as the automatic exposure control (AEC) to determine X-ray energy and flux intensities required to optimize the image quality. Phantom sizes with dimensions $115 \mathrm{~mm}$ x $79 \mathrm{~mm}$ x $92 \mathrm{~mm}$ $(\mathrm{L} \times \mathrm{W} \times \mathrm{H})$ presented a reasonable volume for imaging to the respective modalities and also helped to avoid edge-effects when assessing the appearance of the renal 205 arteries in the images.

Glass beads (Crown Jewels, Dublin, Ireland) with a diameter of $4 \mathrm{~mm}$ were chosen as suitable fiducial markers to facilitate image registration across the modalities. Using sewing thread (diameter $0.8 \mathrm{~mm}$ ), four rows of four beads were threaded through the 210 phantom, with two rows of beads located on either side of the renal artery lumen. On each row the beads were separated from each other by $2.8 \mathrm{~mm}$ and secured with adhesive. The bottom row was secured $57 \mathrm{~mm}$ from the top of the container and 20 $\mathrm{mm}$ from the edges of the container. The upper row of each pair was placed $8 \mathrm{~mm}$ above the first and $10 \mathrm{~mm}$ from the edge of the container. This lateral displacement

215 of the top rows relative to the position of the lower rows was used to minimize any obstruction of the lower row caused by shadowing in the US images deriving from the upper row. The glass bead marker system is presented in Figure 3. 

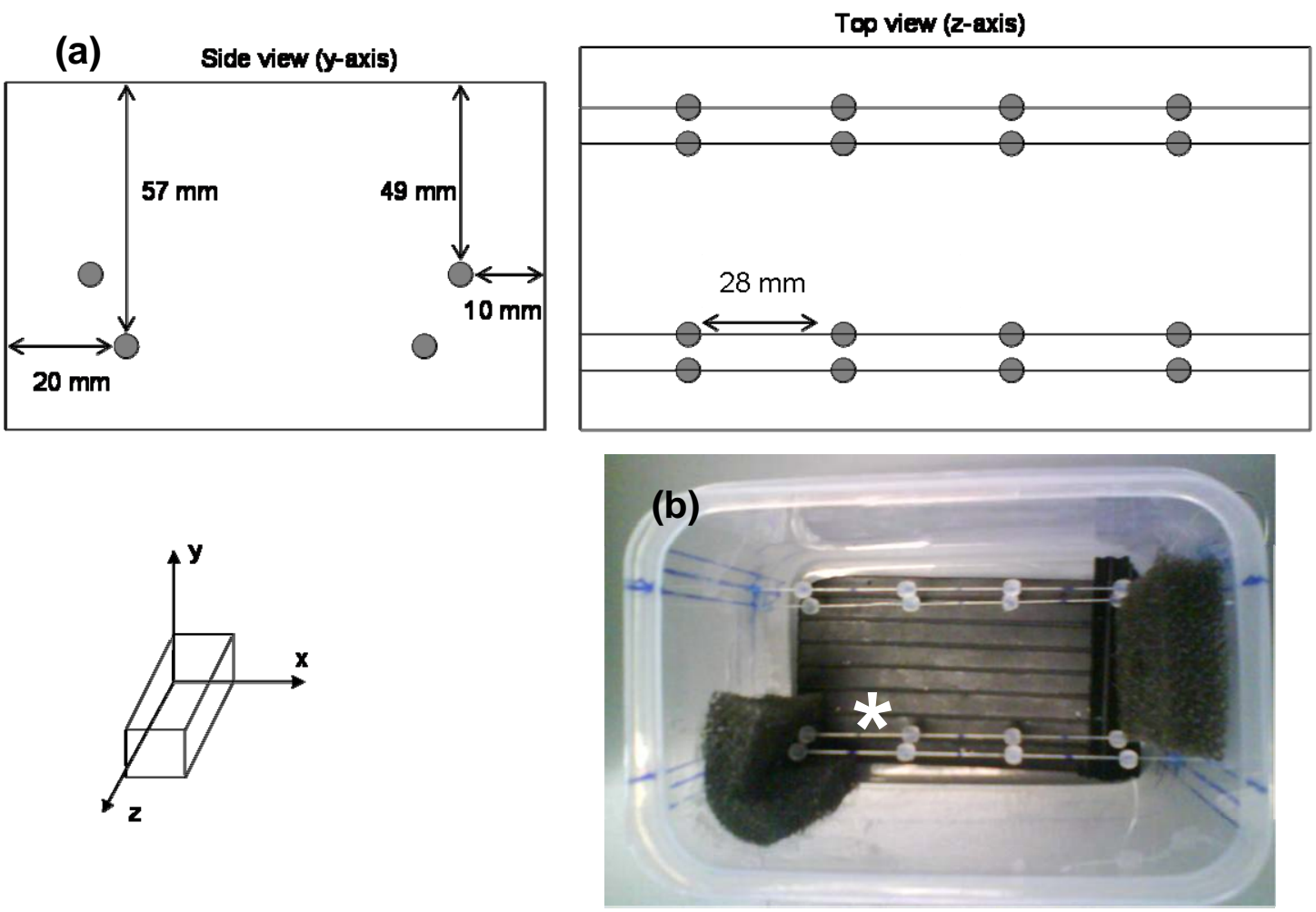

220 Figure 3 (a) Cross-sectional schematic diagram of the fiducial markers in the phantoms, (b) Photograph showing the positioning of the glass bead markers before inclusion of the metal artery model and background TMM, where * marks the location of the stenosis relative to the fiducial markers.

\section{Image acquisition of phantoms}

The phantoms were imaged with standard clinical imaging systems, using basic imaging protocols rather than specific clinical protocols or flow measurements, since the aim of the study was to assess the visualization of the phantoms with each imaging modality and determine the respective geometric accuracies. The phantoms were imaged with no liquid in the vessels for CT and MRI, with water in the vessels for US, and with a water-contrast agent mixture for DSA. Longitudinal and transverse B-mode ultrasound images were acquired with a HDI 3000 system (Philips Medical Systems, Netherlands) using a linear array transducer (L12-5) at a nominal frequency of $6 \mathrm{MHz}$. The images were optimized in each case with the output power set at maximum, focal position at the centre of the vessel and a field of view of $8 \mathrm{~cm}$. 
3D $T_{2}$-weighted MR images were acquired with a 3T Philips Achieva system (Philips Medical Systems, Netherlands) using a turbo spin echo (TSE) technique, with the phantoms placed inside an 8-channel phased array detector coil (Philips Head SENSE 8). Images were obtained using the following acquisition parameters: resolution $=0.8 \times 0.8 \times 0.8 \mathrm{~mm}$, field of view $=150 \times 141 \times 130 \mathrm{~mm}$, time to echo $(\mathrm{TE})=380 \mathrm{~ms}$, time to repetition $(\mathrm{TR})=2500 \mathrm{~ms}$, Turbo factor $=120$, number of signal averages $=2$, SENSE parallel imaging factor $=2 . \mathrm{CT}$ images were acquired with a GE Discovery STE system (GE Healthcare, UK) which included a full GE light speed helical 16-Slice CT using a slice thickness of $0.6 \mathrm{~mm}$, a scan range of $245120 \mathrm{~mm}$, and a matrix size of $1024 \times 1024$. The current was $250 \mathrm{~mA}$ and peak voltage was $120 \mathrm{kV}$. The computed tomography dose index (CTDI) was $35.52 \mathrm{mGy}$ and dose length product (DLP) was 572.2 mGy.cm. DSA images were acquired on a Philips Integris Allura System, (Philips Medical Systems, Netherlands), with each vascular phantom filled initially with water and then with a contrast solution of

250 Ultravist (Bayer Schering Pharma AG, Germany) at $240 \mathrm{mg} \mathrm{ml}^{-1}$. The concentration of the contrast agent was carefully selected to mimic the image intensity enhancement obtained clinically. Projections were obtained parallel to the vessel using the following parameters: source-to-detector distance $=67 \mathrm{~cm}$, field-of-view $=23 \mathrm{~cm}$, table height $99 \mathrm{~cm}$, current $=400 \mathrm{~mA}$ and peak voltage $=50 \mathrm{kV}$. Each image 255 sequence was captured at a frame rate of 6 frames per second with $4 \mathrm{~ms}$ of exposure with a matrix size of $1024 \times 1024$.

\section{Diameter measurements}

To determine the spatial accuracy of each imaging modality, the diameter of the vessel lumen was measured at three locations in the respective images of each 
phantom $(0,30,50,70$ and $85 \%$ stenosis $)$; these locations are illustrated in Figure 4 (position $\mathrm{A}$ - before stenosis: $10 \mathrm{~mm}$ from inlet; position $\mathrm{B}$ - at centre of stenosis: $15 \mathrm{~mm}$ from inlet; and position $\mathrm{C}$ - after stenosis: $10 \mathrm{~mm}$ from bend). These measurements were compared with reference measurements of the diameter of the 265 metal model used to produce the lumen in each phantom, which were measured five times at each location using a Vernier caliper. A one way ANOVA statistical test was carried out to compare the vessel lumen diameters determined from each imaging modality. The null hypothesis being tested was that there was no difference in the dimensions of the models. If $\mathrm{p}<0.05$, the null hypothesis was rejected and there was

270 a statistical difference in the dimensions of the models. All statistical tests were carried out using Minitab ${ }^{\circledR}$ Statistical Software (version 15).

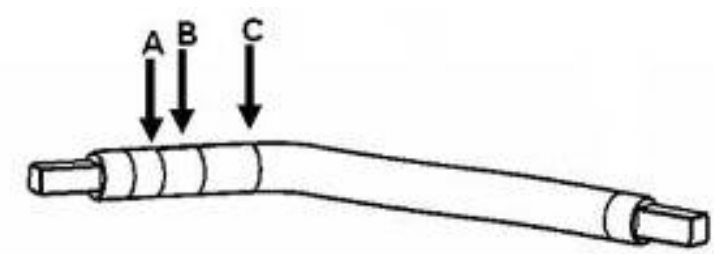

Figure 4 Location of the reference measurements on the metal model at position A-before stenosis: $10 \mathrm{~mm}$ from inlet; position B- at stenosis: $15 \mathrm{~mm}$ from inlet; and position $\mathrm{C}$ - after 275 stenosis: $10 \mathrm{~mm}$ from bend.

Transverse B-mode was used to determine the diameter of the vessels in the US images. For MRI and CT images, the fiducial markers were used to identify and orientate the plane views, and the coronal slices containing the mid-point of the vessel 280 for each phantom were selected. For DSA, the diameter was calculated from the subtracted contrast images for each phantom. All of the acquired images were analyzed using a MATLAB (The Mathworks, USA) program developed in-house. This program allowed for a line profile to be drawn across the lumen extending over 
the vessel-lumen boundaries at each position, from which the vessel lumen diameter could be determined by plotting the image intensity versus distance across each region for all modalities; an example is illustrated in Figure 5 for a transverse B-mode US image. A graphical interface in the MATLAB program was used to display the intensity profile along the line of interest, allowing the user to specify the beginning and end of the vessel. The peaks corresponding to the edges of the near and far walls were located. The distance in [mm] was calculated by measuring the known dimensions of the phantom and converting the pixel distance in $\mathrm{mm}$.
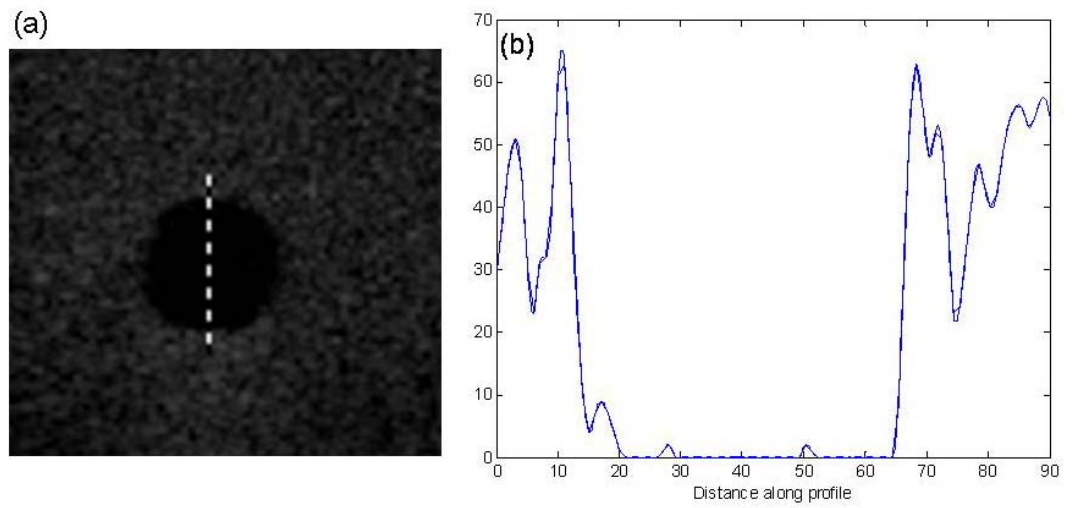

Figure 5 Calculation of the vessel diameter in a B-mode ultrasound image: (a) line profile marked across the lumen, (b) plot of image intensity versus distance along the chosen line profile. Reproduced with permission [15]

\section{RESULTS}

\section{TMM Characterization}

300 The measured TMM properties and average in vivo properties for soft tissue in the vicinity of the renal artery are presented in Table 2 . In general, the renal artery is surrounded by fatty tissue which can typically be of the order of $1-2 \mathrm{~cm}$ thick, increasing for obese patients. Beyond the fat layer, a range of other soft tissues are found, for example muscle, pancreases, duodenum (around the right RA), together 305 with several blood vessels (e.g. renal vein, aorta, inferior vena cava). The TMM had 
similar acoustic properties and Hounsfield Number to that of the surrounding soft tissues. The X-ray transmission through the TMM compared closely with that through the water phantom $(<10 \%$ difference between the transmission through both materials, except at very low energies at $50 \mathrm{kVp}$ where the difference was $\sim 15 \%$ ). $\mathrm{T}_{1}$

310 values for the soft tissue surrounding the RA range from $382 \pm 13 \mathrm{~ms}$ for fat to $1545 \pm$ $142 \mathrm{~ms}$ for the kidney medulla [16], so the values measured for the TMM in the current study $(1504 \pm 10 \mathrm{~ms})$ are at the upper bounds of this range. The $\mathrm{T}_{2}$ value of the TMM $(40.0 \pm 0.4 \mathrm{~ms})$, on the other hand, was fairly typical of those values occurring for the soft tissue surrounding the RA, which typically range 315 from $29 \pm 4 \mathrm{~ms}$ for muscle to $81 \pm 8 \mathrm{~ms}$ for the kidney medulla [16].

Table 2 Comparison of average in vivo soft tissue and measured TMM properties

\begin{tabular}{l|c|c}
\hline \hline Parameter & $\begin{array}{c}\text { Average soft tissue } \\
\text { in vivo values }\end{array}$ & $\begin{array}{c}\text { TMM Measured } \\
\text { values }\end{array}$ \\
\hline Speed of sound $\left(\mathrm{m} \mathrm{s}^{-1}\right)$ & 1540 & $1549 \pm 5$ \\
Attenuation coefficient $\left(\mathrm{dB} \mathrm{cm}^{-1} \mathrm{MHz}^{-1}\right)$ & $0.5-0.7$ & $0.52 \pm 0.03$ \\
$\mathrm{~T}_{1}(\mathrm{~ms})$ & $382-1545$ & $1504 \pm 10$ \\
$\mathrm{~T}_{2}(\mathrm{~ms})$ & $29-81$ & $40.0 \pm 0.4$ \\
Hounsfield Number & $20-70$ & $58.6 \pm 7.3$ \\
\hline
\end{tabular}

\section{Visualization of the fiducial markers}

320 Images of the fiducial markers obtained with the four imaging modalities are presented in Figure 6. In each case, the fiducial markers were clearly identifiable and did not introduce artifacts into the images. For US (Figure 3(a)) the glass beads appeared as bright spots in the B-mode images, as the glass had a much greater acoustic impedance $\left(\mathrm{z}=12.1 \times 10^{6} \mathrm{~kg} \mathrm{~m}^{-2} \mathrm{~s}^{-1}\right)$ [17] compared to the surrounding

325 TMM $\left(\mathrm{z}=1.6 \times 10^{6} \mathrm{~kg} \mathrm{~m}^{-2} \mathrm{~s}^{-1}\right)$ (calculated acoustic impedance $(\mathrm{z})=\rho c$ where $\rho$ density $=1049 \mathrm{~kg} \mathrm{~m}^{-3}$ and $c=1549 \mathrm{~m} \mathrm{~s}^{-1}$ [12]). In MRI, the contrast in the 
$\mathrm{T}_{2}$-weighted images stems from the $\mathrm{T}_{2}$ relaxation times of the phantom constituents (TMM and glass beads): since the glass beads have an extremely short $\mathrm{T}_{2}$ value $(<100 \mu \mathrm{s})$, they effectively produce no signal in a $\mathrm{T}_{2}$-weighted image and 330 consequently appear dark, as shown in Figure 3(b). For the imaging techniques based on X-ray transmission (DSA and CT), the image contrast stems from a difference in the X-ray linear attenuation coefficients of the different phantom constituents.

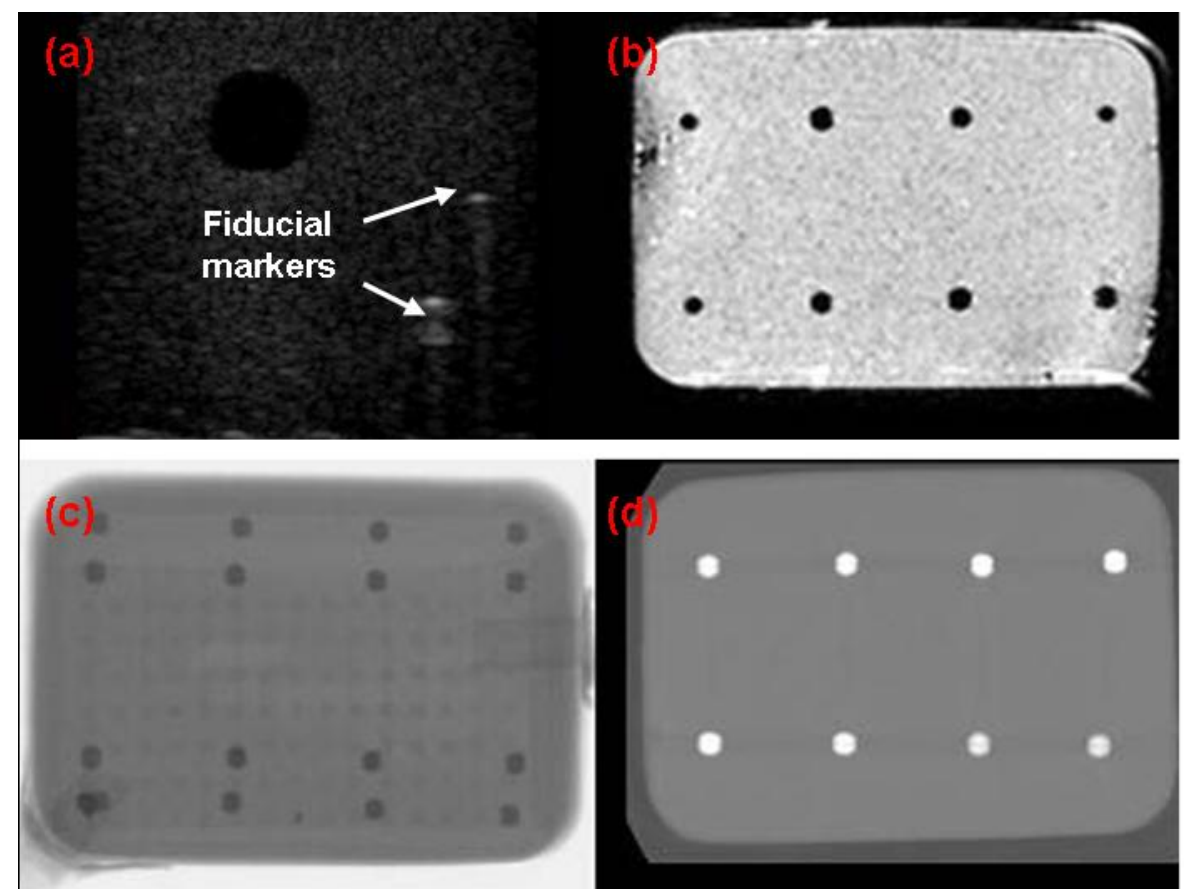

335 Figure 6 Fiducial markers are clearly identifiable in all the imaging modalities (a) ultrasound, transverse image showing cross section of the lumen and 2 beads at depth $49 \mathrm{~mm}$ and $57 \mathrm{~mm}$ on the right side of the phantom, (b) MRI showing a slice with 2 rows of beads, (c) angiographic image and (d) CT showing a slice with 2 rows of beads 


\section{Visualization of renal artery in phantoms}

The images obtained using each imaging modality for the set of phantoms are

345 presented in Figures 7 - 9. Figure 7 shows the 2D B-mode longitudinal US images of the inlet of each phantom. An example of the 2D transverse B-mode images captured for three slices through the phantom with a $50 \%$ stenosis is shown in Figure 8. Presented in Figure 9 are the images of the 5 multi-modality phantoms with varying degrees of stenosis acquired using MRI, CT and angiography contrast images. As can

350 be seen from these images, the vessel of each phantom is clearly visualized and identifiable. 


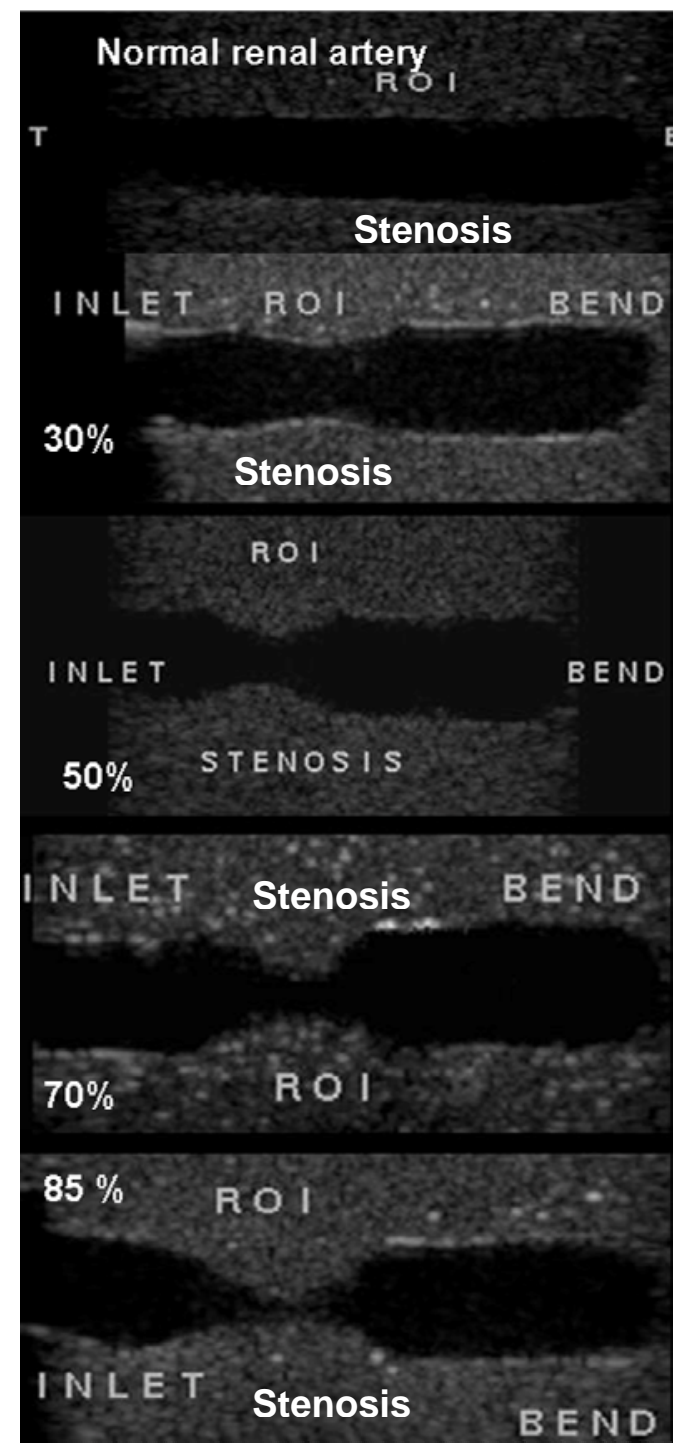

Figure 7 B-mode images showing inlet of the renal artery flow phantom with varying degrees of stenosis $(0,30,50,70$ and $85 \%)$. Reproduced with permission [15]
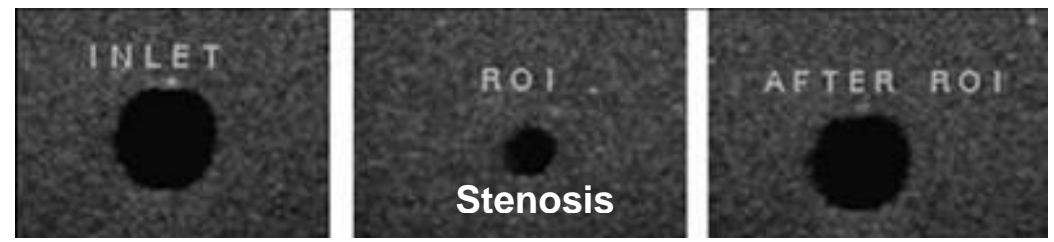

Figure 8 Transverse views of a phantom with a $50 \%$ stenosis at the position of (a) $10 \mathrm{~mm}$ after the inlet, (b) the stenosis and (c) $10 \mathrm{~mm}$ before the bend. 


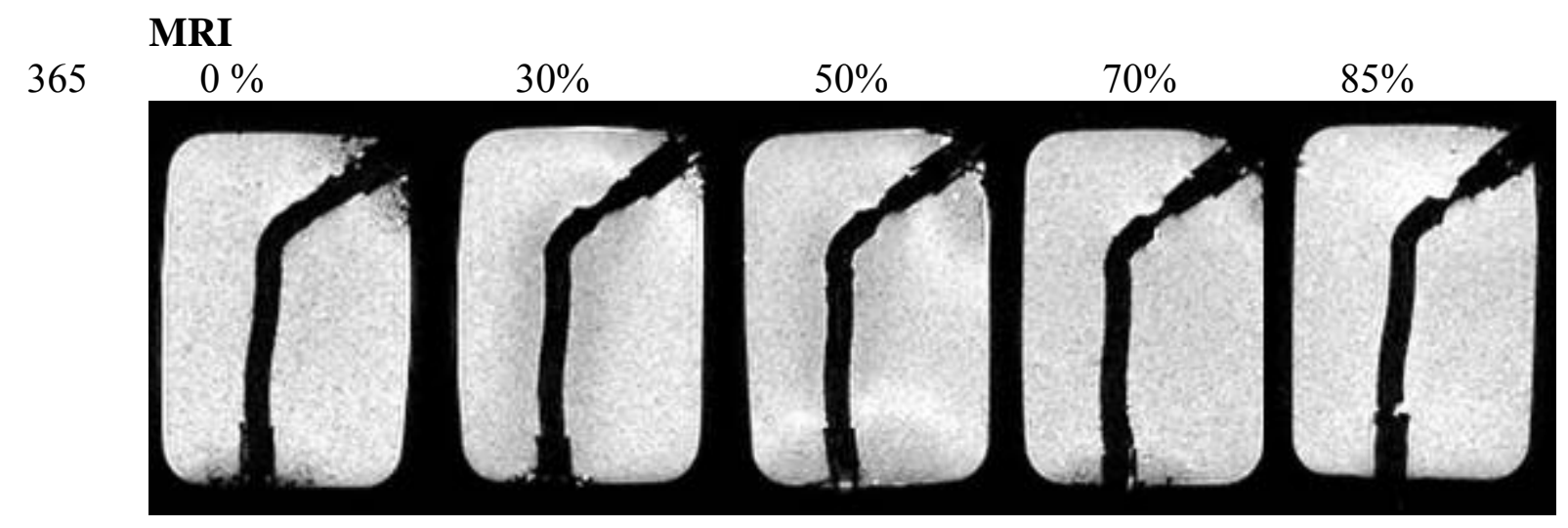

CT

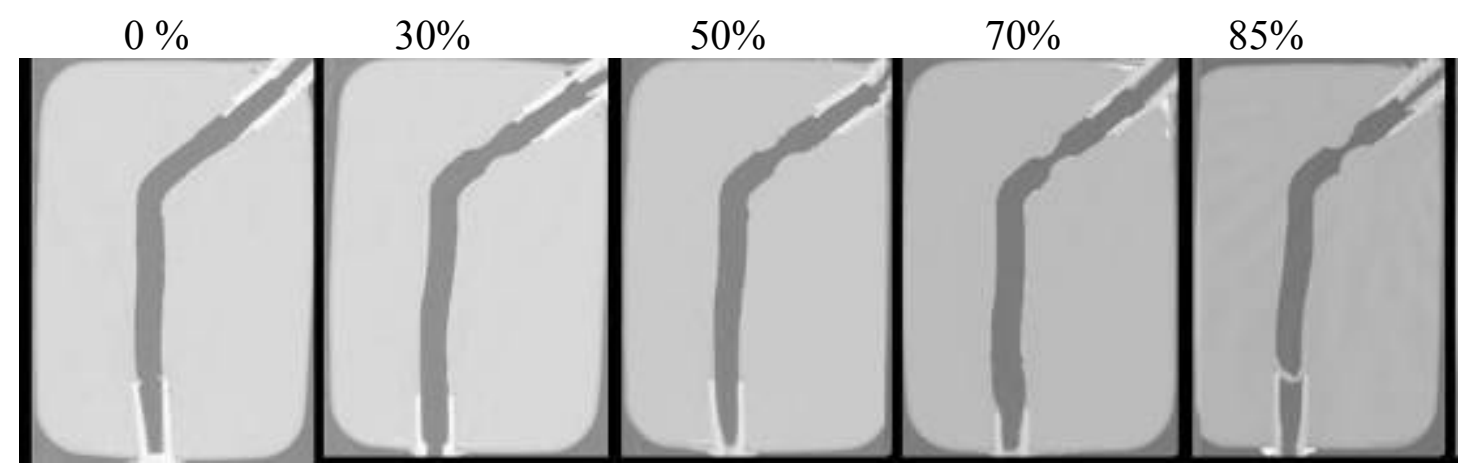

370

\section{DSA}

$0 \%$

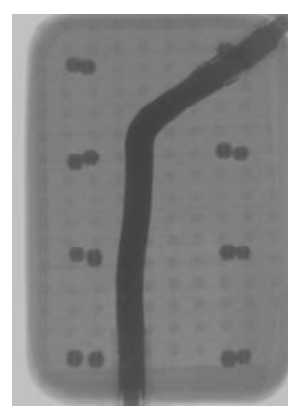

$30 \%$

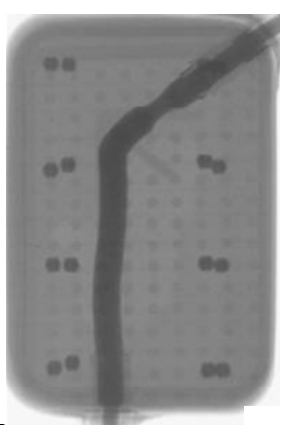

$50 \%$

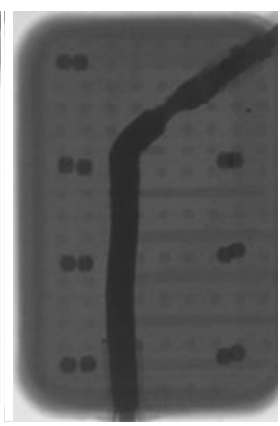

$70 \%$

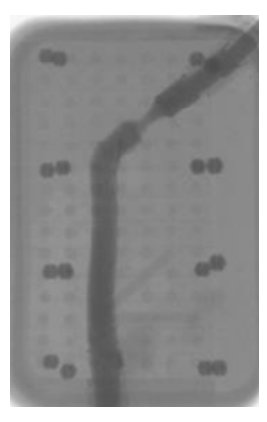

$85 \%$

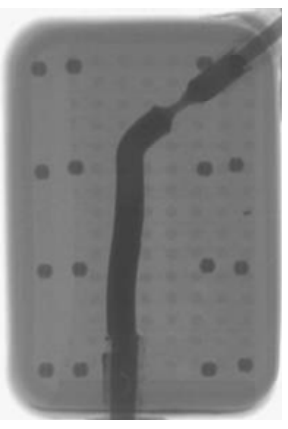

Figure 9 MRI, CT and unsubtracted angiography contrast images of the range of multimodality phantoms

\section{Diameter measurements}

Previous experiments with the production of the metal models used to create the lumens in the phantoms demonstrated the reproducibility of the technique, with little variation in model diameters $[15,18]$. The diameter of the vessel in each phantom was measured from images acquired from each imaging modality. For ultrasound, the 
diameters were calculated from the $2 \mathrm{D}$ transverse images such as those shown in Figure 8. For both MRI and CT, the diameter was calculated from single slices extracted from their acquired volumes at the mid-point of the vessel, examples of which are shown in Figure 9. For DSA, the diameter was calculated from the subtracted contrast images for each phantom, again as shown in Figure 9. In accordance with clinical practice, based on the dimensions taken from the 2D projection, the degree of stenosis in an artery is defined as;

$$
(1-L / R) \times 100
$$

\section{Equation 1}

where $L$ is the diameter of the lumen at the site of the stenosis and $R$ the reference site diameter, representing the diameter of the lumen pre-stenosis. Using this formula the percentage stenosis was calculated for the reference and for each imaging technique, where $L$ was taken to be the diameter at location B (at the stenosis) and $R$ the average diameter from locations A (10 $\mathrm{mm}$ before the stenosis) and C (10) $\mathrm{mm}$ after the stenosis. From these measurements, the percentage error for each degree of stenosis was calculated for each imaging modality. A positive error corresponds to an overestimation in the measurement of the diameter and a negative value an underestimation error in the measurement of the diameter. A summary of the over/under estimation of the degree of stenosis (\%) with US, MRI, CT and DSA is presented in Table 3. 
Table 3 Summary of over/under estimation of stenosis (\%) with US, MRI, CT and DSA

\begin{tabular}{lccccc}
\hline & $\mathbf{0 \%}$ & $\mathbf{3 0 \%}$ & $\mathbf{5 0 \%}$ & $\mathbf{7 0 \%}$ & $\mathbf{8 5 \%}$ \\
\hline US & 0 & $-7^{*}$ & -2 & $-10^{*}$ & $-12^{*}$ \\
MRI & 2 & -3 & -3 & $-5^{*}$ & $-10^{*}$ \\
CT & 3 & $-6^{*}$ & -2 & $-10^{*}$ & $-13^{*}$ \\
DSA & $6 *$ & 3 & $-7^{*}$ & $-12^{*}$ & $-14^{*}$ \\
\hline P value & 0.0045 & $<0.0001$ & 0.0110 & $<0.0001$ & $<0.0001$ \\
\hline \hline
\end{tabular}

\section{DISCUSSION}

410

The use of rapid prototyping techniques in the fabrication of the anatomically-realistic renal arteries significantly reduces the time and cost involved in making a range of phantoms replicating different grades of RAS since only one master silicon mould is required, which can be reused repeatedly to produce various grades of stenosis with

415 the use of the variable diameter inserts. In previous studies, separate master moulds were required to produce the different grades of stenosis in each individual phantom $[19,20]$. The TMM chosen for the phantoms was developed as part of the European Commission-funded project [12] and is the IEC-recommended TMM for use in ultrasound test phantoms [21]. This TMM has been reported to have acoustic

420 properties similar to soft tissue over the frequency range 2- $20 \mathrm{MHz}[12,14,22]$. To the authors' knowledge, this TMM has not previously been used for modalities other than ultrasound, and thus it is of interest to characterize the MR and X-ray imaging properties, particularly the Hounsfield Number of this material in order to determine its suitability across the imaging modalities. The fact that these properties replicated

425 closely those found in vivo for soft tissues in the vicinity of the renal artery gives confidence in its use as a multi-modality TMM. The $\mathrm{T}_{1}$ relaxation value, which was at the upper bounds of that found in vivo, could be easily reduced through the addition 
of a paramagnetic relaxation agent such as $\mathrm{MnCl}_{2}$, which is common practice in the production of phantoms for MR applications. The X-ray attenuation of the TMM was not determined as part of this study as a calibrated monoenergetic X-ray source was not available to carry out this measurement, however, the Hounsfield Number of the TMM was determined and found to be similar to the average value for soft tissue.

All of the renal artery phantoms were successfully imaged with the four imaging modalities, demonstrating the potential of this phantom as a standard multi-modality vascular test tool. Furthermore, the glass beads used as fiducial markers were clearly identifiable and did not introduce artifacts into the images. The measured diameters of the imaged stenoses were determined using a relatively simple $2 \mathrm{D}$ approach and compared to the known values, with the most accurate technique found to be MRI, 440 followed by US, CT and DSA. The reader is referred to Hoffmann et al. for a discussion of more complex methods for determining vessel diameters in DSA images, which has been found to improve the accuracy of this technique [23]. There was a general trend for the modalities to perform worse as the degree of stenosis increased. For the higher stenosed phantoms $(70 \%$ and $85 \%)$, all four imaging modalities significantly underestimated the stenosis, with DSA having the greatest underestimation. The accuracy of US for stenoses $<50 \%$, with mean underestimation of $3.5 \%$ was comparable to results reported in the literature $[24,25] . \quad$ It has been shown previously that DSA tends to underestimate diameters [25] and this may be because it uses a limited number of projections and can therefore fail to detect the maximum stenosis in the artery. Unlike DSA, a 3D volume acquisition technique was used for the MRI data, while CT acquires projections at different angles, followed by filtered backprojection image reconstruction, which reduces the partial volume effects 
compared to the simple DSA approach which can contain pixels with a mixture of tissue attenuations. The image processing technique of maximum intensity projection which is commonly used in CT produces an overview of the target vessel which aids the detection of the maximum extent of the stenosis in the vessel. MRI was the most accurate imaging modality with a mean underestimation of $4 \%$, while both US and CT had comparable results.

460 The aim of the current study was to demonstrate the potential of the anatomicallyrealistic renal artery phantoms for comparative studies involving US, MRI, CT and DSA, and therefore default image acquisition parameters were used rather than making use of pre-set values typically used for specific anatomical regions, which might have proved inappropriate for the relatively small phantom used in the current 465 study. Clinically, Spectral Doppler is the most common ultrasound mode for calculating the degree of renal artery stenosis based on velocity measurements in the vicinity of the stenosis, while MRA and CTA are increasingly being used to image renal artery stenosis. The phantoms were primarily designed as flow phantoms, and further investigation will focus on their use in this capacity with clinical protocols 470 using Spectral Doppler US, MR Angiography (MRA) and CT Angiography (CTA) techniques.

Although the current study allowed for a standardized comparison between the four imaging techniques to be carried out in a highly controlled manner, nevertheless a 475 limitation was the use of relatively simple symmetrical stenoses of the same length. Clinically, stenoses are more complex and present in a variety of lengths and eccentricities, and furthermore contain fatty and/or calcified material deposited in an 
asymmetrical manner in the vessel wall and within the lumen itself. Further work by the authors is focusing on the development of more complex phantoms incorporating a vessel wall mimic, together asymmetrical stenoses incorporating fatty-mimicking and calcified material, and a pump system producing a pulsatile flow to allow for the study of the haemodynamic properties of the renal artery system using the different imaging modalities.

\section{CONCLUSIONS}

A range of multi-modality anatomically-realistic renal artery phantoms were 490 developed and artifact-free images of the phantoms were obtained using the four techniques currently used to detect renal artery stenosis (US, MRI, CT and X-ray DSA). The TMM used in the phantoms' construction was found to have US, MR and CT properties comparable to in vivo soft tissue, while the phantoms' layout proved suitable for imaging with the various modalities. The severity of stenosis was underestimated in all cases, with the degree of underestimation greatest for the $70 \%$ and $85 \%$ stenosed phantoms, with DSA performing consistently worse than the other imaging modalities. This study has demonstrated the potential of these novel phantoms to be used as a standard multi-modality vascular phantom for the evaluation of RAS-diagnosis techniques,

\section{ACKNOWLEDGEMENTS}

This work was supported by the Technological Sector Research Strand 1 Scheme, HEA and the TERs 2005 and CABS 2007 Schemes, Research Support Unit of the Dublin Institute of Technology. C. M. Moran would like to acknowledge funding from the British Heart Foundation grant PG/07/107/23895. 


\section{REFERENCES}

1. Bosmans, J.L. and M.E. De Broe, Renovascular hypertension: diagnostic and

2.

2. Zalunardo, N. and K.R. Tuttle, Atherosclerotic renal artery stenosis: current status and future directions. Curr Opin Nephrol Hypertens, 2004. 13(6): p. 613-21.

3. Olin, J.W., et al., The utility of duplex ultrasound scanning of the renal arteries for diagnosing significant renal artery stenosis. Ann.Intern.Med., 1995. 122(11): p. 833-838.

4. Radermacher, J. and R. Brunkhorst, Diagnosis and treatment of renovascular stenosis--a cost-benefit analysis. Nephrol.Dial.Transplant., 1998. 13(11): p. 2761-2767.

$520 \quad 5 . \quad$ Reynolds, H.R., et al., Frequency of severe renal artery stenosis in patients with severe thoracic aortic plaque. Am J Cardiol, 2004. 94(6): p. 844-6.

6. Smith, R.F., et al., Geometric characterization of stenosed human carotid arteries. Acad Radiol, 1996. 3(11): p. 898-911.

7. Cloutier, G., et al., A multimodality vascular imaging phantom with fiducial 525 markers visible in DSA, CTA, MRA, and ultrasound. Med Phys, 2004. 31(6): p. 1424-33.

8. Dabrowski, W., et al., A real vessel phantom for imaging experimentation. Med Phys, 1997. 24(5): p. 687-93.

9. Frayne, R., et al., A geometrically accurate vascular phantom for comparative studies of $x$-ray, ultrasound, and magnetic resonance vascular imaging: construction and geometrical verification. Med Phys, 1993. 20(2 Pt 1): p. 41525.

10. Meagher, S., et al., Anatomical flow phantoms of the nonplanar carotid bifurcation, part II: experimental validation with Doppler ultrasound. Ultrasound Med Biol, 2007. 33(2): p. 303-10.

11. Allard, L., et al., Multimodality vascular imaging phantoms: a new material for the fabrication of realistic 3D vessel geometries. Med Phys, 2009. 36(8): p. 3758-63.

12. Teirlinck, C.J., et al., Development of an example flow test object and comparison of five of these test objects, constructed in various laboratories. Ultrasonics, 1998. 36(1-5): p. 653-60.

13. Ramnarine, K.V., T. Anderson, and P.R. Hoskins, Construction and geometric stability of physiological flow rate wall-less stenosis phantoms. Ultrasound Med Biol, 2001. 27(2): p. 245-50.

545 14. Browne, J.E., et al., Assessment of the acoustic properties of common tissuemimicking test phantoms. Ultrasound Med Biol, 2003. 29(7): p. 1053-60.

15. King, D.M., et al., Development of a range of anatomically realistic renal artery flow phantoms. Ultrasound Med Biol, 2010. 36(7): p. 1135-44.

16. de Bazelaire, C.M., et al., MR imaging relaxation times of abdominal and pelvic tissues measured in vivo at 3.0 T: preliminary results. Radiology, 2004. 230(3): p. 652-9.

17. Selfridge, A.R., Approximate material properties in isotropic materials. IEEE Trans Sonics Ultrasound, 1985. SU-32: p. 13.

18. King, D.M., Development of renal phantoms for the evaluation of current and emerging ultrasound technology, in School of Physics. 2009, Dublin Institute of Technology: Dublin. 
19. Poepping, T.L., et al., An in vitro system for Doppler ultrasound flow studies in the stenosed carotid artery bifurcation. Ultrasound Med Biol, 2002. 28(4): p. 495-506.

560 20. Watts, D.M., et al., Anatomical flow phantoms of the nonplanar carotid bifurcation, part I: computer-aided design and fabrication. Ultrasound Med Biol, 2007. 33(2): p. 296-302.

21. IEC, Ultrasonics - Flow measurement systems: Flow test object, G. International Electrotechnical Commission, Switzerland, Editor. 2001.

565 22. Brewin, M.P., et al., The acoustic properties, centered on $20 \mathrm{MHZ}$, of an IEC agar-based tissue-mimicking material and its temperature, frequency and age dependence. Ultrasound Med Biol, 2008. 34(8): p. 1292-306.

23. Hoffmann, K.R., et al., Vessel size measurements in angiograms: a comparison of techniques. Med Phys, 2002. 29(7): p. 1622-33.

570 24. Beux, F., et al., Automatic evaluation of arterial diameter variation from vascular echographic images. Ultrasound Med Biol, 2001. 27(12): p. 1621-9.

25. Boussion, N., et al., Geometrical accuracy and fusion of multimodal vascular images: a phantom study. Med Phys, 2004. 31(6): p. 1434-43. 